\title{
High-Resolution MRI Vessel Wall Imaging: Spatial and Temporal Patterns of Reversible Cerebral Vasoconstriction Syndrome and Central Nervous System Vasculitis
}

\author{
E.C. Obusez, F. Hui, R.A. Hajj-ali, R. Cerejo, L.H. Calabrese, T. Hammad, and S.E. Jones
}

\begin{abstract}
BACKGROUND AND PURPOSE: High-resolution MR imaging is an emerging tool for evaluating intracranial artery disease. It has an advantage of defining vessel wall characteristics of intracranial vascular diseases. We investigated high-resolution MR imaging arterial wall characteristics of CNS vasculitis and reversible cerebral vasoconstriction syndrome to determine wall pattern changes during a follow-up period.
\end{abstract}

MATERIALS AND METHODS: We retrospectively reviewed 3T-high-resolution MR imaging vessel wall studies performed on 26 patients with a confirmed diagnosis of CNS vasculitis and reversible cerebral vasoconstriction syndrome during a follow-up period. Vessel wall imaging protocol included black-blood contrast-enhanced T-weighted sequences with fat suppression and a saturation band, and time-of-flight MRA of the circle of Willis. Vessel wall characteristics including enhancement, wall thickening, and lumen narrowing were collected.

RESULTS: Thirteen patients with CNS vasculitis and 13 patients with reversible cerebral vasoconstriction syndrome were included. In the CNS vasculitis group, 9 patients showed smooth, concentric wall enhancement and thickening; 3 patients had smooth, eccentric wall enhancement and thickening; and 1 patient was without wall enhancement and thickening. Six of 13 patients had follow-up imaging; 4 patients showed stable smooth, concentric enhancement and thickening; and 2 patients had resoluton of initial imaging findings. In the reversible cerebral vasoconstriction syndrome group, 10 patients showed diffuse, uniform wall thickening with negligible-to-mild enhancement. Nine patients had follow-up imaging, with 8 patients showing complete resolution of the initial findings.

CONCLUSIONS: Postgadolinium 3T-high-resolution MR imaging appears to be a feasible tool in differentiating vessel wall patterns of CNS vasculitis and reversible cerebral vasoconstriction syndrome changes during a follow-up period.

ABBREVIATIONS: HRMRI = high-resolution MR imaging; RCVS = reversible cerebral vasoconstriction syndrome

$\mathbf{R}$ eversible cerebral vasoconstriction syndrome (RCVS) and CNS vasculitis are 2 distinct cerebrovascular disease entities with overlapping presenting symptoms of headache and, not uncommonly, neurologic deficits, which may be secondary to ischemic and/or hemorrhagic stroke. ${ }^{1-4}$ Expeditious diagnosis is critical to discriminate the 2 disease entities to initiate appropriate and timely treatment. ${ }^{4}$ In addition to clinical and laboratory work-up, DSA, MRA, and CTA are the preferred imaging modalities, essential to diagnosis and disease management. However, current vascular imaging fails to

\footnotetext{
Received September 13, 2013; accepted after revision January 10, 2014.

From the Department of Diagnostic Radiology (E.C.O., S.E.J.), Imaging Institute; Cerebrovascular Center (F.H.); Department of Neurology (R.A.H., R.C.), Neurological Institute; and Department of Rheumatology (L.H.C., T.H.), Orthopaedic and Rheumatology Institute, Cleveland Clinic, Cleveland, Ohio.

Please address correspondence to Stephen E. Jones, MD, PhD, Department of Diagnostic Radiology-Imaging Institute, The Cleveland Clinic, Mail Code U15, 9500 Euclid Ave, Cleveland, OH 44195; e-mail: joness19@ccf.org

三 Indicates article with supplemental on-line tables.

Indicates article with supplemental on-line figures.

http://dx.doi.org/10.3174/ajnr.A3909
}

distinguish both entities, often due to shared nonspecific luminal findings on DSA, MRA, and CTA. ${ }^{1,5}$

Radiographic discrimination using current vascular imaging is difficult due to the relative thinness of intracranial vessel walls, at most, 1-2 mm thick in the largest intracranial vessels. The size scale is near the resolution limit of present imaging technology. While DSA provides superior resolution, it only images the vessel lumen. CTA and MR imaging, on the other hand, can image extraluminal tissue; however, the resolution is near the limit for imaging the vessel wall. Of these methods, MR imaging tends to be the technique of choice due to its superior soft-tissue contrast compared with CTA. Thus, the successful application of MR imaging to scan the vessel wall would require using a higher resolution than is routinely used in clinical practice while using standard clinical imaging hardware. This is accomplished by extending the technical parameters of a clinical MR imaging scanner as much as possible within the limits of the scan time and signal and is termed "high-resolution MR imaging" (HRMRI).

The utility of HRMRI in characterizing vessel wall patterns of 
intracranial artery diseases has been investigated. ${ }^{6-9}$ Recent studies have identified distinct characteristics of arterial wall thickening and wall enhancement in RCVS and CNS vasculitis. ${ }^{6,7}$ While these studies have described spatial patterns on HRMRI, they have not described the temporal evolution of these diseases; this information could further aid diagnosis and management. The aim of this study was to describe the spatial patterns and temporal evolution of RCVS and CNS vasculitis by examining vessel wall characteristics during a follow-up period by using 3T-HRMRI and thereby assess the potential of HRMRI to serve as a surveillance technique to identify changes in wall morphology with disease progression or remission. We hypothesized that 3T-HRMRI vessel wall imaging may differentiate the spatial and temporal patterns of RCVS and CNS vasculitis.

\section{MATERIALS AND METHODS \\ Patients}

This single tertiary center study was approved by the institutional review board at our institution. Patients were identified from an electronic medical data base of 48 patients with intracranial vessel disease who underwent an HRMRI vessel wall imaging protocol between July 2009 and June 2012. Twenty-six patients with a known diagnosis of CNS vasculitis and RCVS were included in the study. Inclusion criteria comprised only patients with an established clinical diagnosis of RCVS and CNS vasculitis who underwent an HRMRI protocol. All diagnoses of RCVS and CNS vasculitis were confirmed to have met established RCVS experience-based guidelines and CNS vasculitis criteria, ${ }^{1,11,12}$ by an expert rheumatologist (R.A.H.) and neuroradiologists (F.H., S.E.J.). A retrospective chart and imaging review was then conducted.

The experience-based guidelines used as criteria for RCVS were first proposed by Calabrese et $\mathrm{al}^{1}$ and were subsequently adopted in the "International Classification of Headache Disorders: 2nd ed." ${ }^{10}$ They include the following: 1) angiography, MRA, or CTA documenting multifocal segmental cerebral artery vasoconstriction; 2) no evidence of aneurysmal subarachnoid hemorrhage; 3 ) normal or near-normal CSF analysis; 4) severe, acute headaches, with or without additional neurologic signs or symptoms; and 5) reversibility of angiographic abnormality within 12 weeks after onset or postmortem examination to rule out vasculitis, intracranial atherosclerosis, and aneurysmal subarachnoid hemorrhage. Four patients had biopsy-proved CNS vasculitis; the remaining 9 patients met established criteria by Calabrese and Mallek ${ }^{11}$ for CNS vasculitis. The criteria include the following: 1) the presence of an acquired, or otherwise unexplained, neurologic deficit after thorough clinical and laboratory evaluation; 2) documentation by cerebral angiography and/or histopathologic features of angiitis within the CNS; and 3) no evidence of systemic vasculitis or any condition with similar angiographic or pathologic features.

\section{Intracranial Vessel Wall Protocol, Sequence, and Acquisition}

All scans were obtained on 3T whole-body scanners (Skyra or Trio; Siemens, Erlangen, Germany) with a 20-channel coil. The protocol started with a standard multislab 3D TOF-MRA sequence centered in the circle of Willis for lumenographic identi- fication of any stenosis. High-resolution imaging used 4 T1weighted spin-echo sequences in the coronal, axial, and sagittal planes. The first was without contrast, followed by 3 with contrast (gadobutrol, Gadovist; Bayer Schering Pharma, Berlin, Germany): 1) a noncontrast $\mathrm{T} 1$ coronal $2-\mathrm{mm}$ section with no gap $(\mathrm{TR} / \mathrm{TE}=$ $11 / 544 \mathrm{~ms})$, matrix $=256 \times 256$, and FOV $=130 \times 130 \mathrm{~mm} ; 2)$ a postgadolinium $\mathrm{T} 1$ coronal $2-\mathrm{mm}$ section with no gap and fat suppression and a saturation band (TR/TE $=11 / 544 \mathrm{~ms})$, matrix $=256 \times 256$, and FOV $=130 \times 130 \mathrm{~mm}$; 3) a postgadolinium $\mathrm{T} 1$ axial 2-mm section with no gap and fat suppression and a saturation band $(\mathrm{TR} / \mathrm{TE}=11 / 750 \mathrm{~ms})$, matrix $=256 \times 256$, and FOV $=130 \times 130 \mathrm{~mm}$; and 4$)$ a postgadolinium sagittal 2-mm section with a $0.4-\mathrm{mm}$ gap and no fat suppression and a saturation band $(\mathrm{TR} / \mathrm{TE}=10 / 600 \mathrm{~ms})$, matrix $=256 \times 256$, and $\mathrm{FOV}=$ $130 \times 130 \mathrm{~mm}$.

The resultant voxel size from the parameters was $0.51 \times$ $0.51 \times 0.2$. The TR/TE parameters provided a black-blood technique for large-to-medium vessels. The protocol was designed for large-vessel evaluation (the middle cerebral arteries, basilar artery, and internal carotid arteries); however, smaller vessels were often interrogated. Due to the small size of each section, it was not practical to cover the entire brain in a clinically acceptable time; therefore, an imaging slab about $2 \mathrm{~cm}$ thick was selected to focus on only the large proximal vessels, with each major vessel viewed in both a longitudinal and transverse direction. If the TOF-MRA showed stenosis outside this default region, the imaging volume was adjusted accordingly or extra slabs were included. The TOFMRA scan parameters were the following: matrix of $384 \times 70$, FOV of $200 \times 150 \mathrm{~mm}$, and section thickness of 0.50 (reconstructed). The resultant voxel sizes from the parameters were $0.74 \times 0.52 \times 0.80$ (acquired) and $0.52 \times 0.52 \times 0.50$ (reconstructed).

\section{Image Analysis}

Images were analyzed and interpreted on an Impax 6.0 PACS. A qualitative examination was initially performed by 2 experienced neuroradiologists (F.H., S.E.J.) blinded to clinical data, who rereviewed stenosis on TOF-MRA and then vessel wall pathology on HRMRI vessel wall images individually and then with consensus reading on the same workstation. They recorded the following properties: the location of pathology, the presence and character of wall thickening (including length and shape, either concentric or eccentric), and the presence and character of wall enhancement. The presence or absence of enhancement was determined by comparing pregadolinium and postgadolinium vessel wall imaging. The strength and pattern of enhancement on the postgadolinium images was considered unequivocal when found in $>1$ imaging plane. If enhancement was present, the strength of the enhancement was classified qualitatively on a 2-point scale as mild or strong and the pattern of enhancement, as concentric or eccentric. We subjectively determined the following on postgadolinium MR imaging; no enhancement if enhancement was absent, mild enhancement if there was thin-wall artery hyperintensity, and strong enhancement if there was thick-wall artery hyperintensity. Concentric enhancement was defined when the artery wall pattern was uniform and circumferential and distributed evenly along entire circumference of the wall. Eccentric enhance- 
ment was defined as clearly nonuniform and noncircumferential, on 1 side of the vessel and not involving the entire circumference. The length of enhancement was measured in millimeters for each lesion by using the utility tool. MRA findings were compared with the HRMRI findings for concordance of abnormalities. The degree of stenosis expressed as a percentage was determined by using North American Symptomatic Carotid Endarterectomy Trial criteria $^{12}$ calculation $[(\mathrm{Da}-\mathrm{Db}) / \mathrm{Da}]$, where $\mathrm{Da}$ is the lumen diameter of a normal vessel and $\mathrm{Db}$ is the diameter of stenosed vessel to the normal vessel. It was determined to be mild if the stenosis was $<10 \%-29 \%$, moderate if between $30 \%$ and $69 \%$, severe if greater than $70 \%-99 \%$, and occluded when a long flow void was seen in the vessel.

\section{Demographic and Clinical Variables}

The collected clinical and demographic data included age, sex, race, date of symptom onset, diagnosis, date of diagnosis, and medications.

\section{RESULTS}

\section{Clinical and Demographic Data}

Twenty-nine patients had an established clinical diagnosis of intracranial disease. Of these, 13 patients were diagnosed with RCVS; and 13 patients, with CNS vasculitis. The median age in the RCVS group was 52 years (range, 15-61 years) with $85 \%$ female, and $9(69 \%)$ patients had follow-up imaging during a median period of 3 months. The median time from presentation to vessel wall imaging for RCVS was 0.5 months (range, 0.2-2.6 months) (On-line Table 1). The median age in the CNS vasculitis group was 42 years (range, $18-69$ years) with 23\% female, and 6 (46\%) patients had follow-up imaging during a median time of 11.5 months. The median time from presentation to vessel wall imaging for CNS vasculitis was 9 months (range, 1.5-171 months).

Disease management in the RCVS group included all patients receiving oral verapamil as treatment until resolution of symptoms or as long-term maintenance therapy (On-line Table 2). In the CNS vasculitis group, initial treatment included an immunosuppressant at diagnosis and maintenance immunosuppressant therapy. Initially, glucocorticoids were used intravenously (highdose methylprednisolone) in 39\% $(n=5)$ of patients, and highdose prednisone was used in $46 \%(n=6)$ of patients. Each patient then received $\geq 1$ dose of other immunosuppressants, which included azathioprine, mycophenolate mofetil, and cyclophosphamide. Seventy-seven percent $(n=10)$ of the patients received steroids for a median of 27 months (range, 5-156 months), 23\% of patients $(n=3)$ received mycophenolate for a median of 10 months (range, 9-96 months), 62\% of patients $(n=8)$ received cyclophosphamide for a median of 5.5 months (range, 1-12 months), and $54 \%$ of patients $(n=7)$ received azathioprine for a median period of 22 months (range, 1-78 months).

\section{HRMRI Vessel Imaging and TOF-MRA Findings}

Intracranial Vasculitis. In $\geq 1$ artery, 9 patients had predominantly smooth, concentric wall enhancement and thickening; 3 patients had smooth, eccentric wall enhancement and thickening; and 1 patient had no wall enhancement or thickening. The diagnosis of CNS vasculitis was confirmed according to the proposed criteria by Calabrese and Mallek. ${ }^{11}$ Sample images of smooth concentric wall enhancement and thickening pregadolinium and postgadolinium are shown in On-line Fig 1. Two of the 9 patients with concentric enhancement had multivessel enhancement. On MRA, these 9 patients revealed varying lumen characteristics of mild-to-severe focal stenosis, diffuse irregular narrowing, and occlusion. Each of these areas of narrowing, stenosis, and occlusion on MRA corresponded to the areas of thickening and enhancement on HRMRI.

A sample HRMRI showing wall enhancement and thickening in $>1$ imaging plane with corresponding MRA stenosis is shown in On-line Fig 2. MRA of 2 of the 3 patients with eccentric enhancement showed no luminal vessel abnormality, while 1 patient had narrowing and stenosis. The 1 patient without arterial wall thickening and enhancement on HRMRI had no luminal abnormality on corresponding MRA. For all 13 patients, the median length of enhancement was $6.1 \mathrm{~mm}$ (range, 3-14 mm). These enhancing segments were mostly unilateral and localized to the anterior cerebral artery, middle cerebral artery, supraclinoid ICA, and terminal ICA. These findings were less frequent in the posterior cerebral artery, basilar artery, and the vertebral artery.

Six patients had follow-up HRMRI, as summarized in On-line Table 2. The median length of enhancement for these 6 patients was $10.8 \mathrm{~mm}$ (range, $2.4-13 \mathrm{~mm}$ ). Of these, 2 patients showed resolution of enhancement, while the remaining 4 patients showed stable but persistent smooth, concentric wall enhancement and thickening during a median follow-up period of 13.5 months (range, 11-16 months). MRA of these 4 patients showed 1 patient with resolution of the initial stenosis and 3 patients with stable narrowing and stenosis. The 2 patients with resolution of smooth, concentric wall enhancement and thickening were reimaged at 7 months and 7.5 months (Fig 1). On MRA, one patient had complete resolution while the other patient had near resolution of initial stenosis.

\section{Reversible Cerebral Vasoconstriction Syndrome}

Thirteen patients were diagnosed with RCVS by using RCVS criteria. ${ }^{1,10}$ The arterial wall findings on initial high-resolution imaging included 10 patients with diffuse, uniform wall thickening, of which 4 showed mild enhancement. Three patients had no arterial wall abnormality. These patterns were found mostly in a bilateral multivessel distribution (Fig $2 A,-B$ and On-line Fig 3A). All 10 patients with diffuse uniform wall thickening, with or without enhancement, had corresponding areas of diffuse irregular narrowing and/or stenosis on MRA (Fig 2E and On-line Fig 3C), except 1 patient with normal vessel findings. The 2 patients with no vessel wall abnormality had no abnormal MRA luminal findings.

Nine of the 13 patients had follow-up imaging, of these, 8 showed resolution of wall thickening and enhancement after 3.5 months, with 1 patient with near-complete resolution with minimal residual wall thickening (Fig $2 D,-E$ and On-line Fig $3 B$ ). One patient remained with diffuse uniform wall thickening and mild concentric enhancement in 1 of the 3 vessels with narrowing on initial imaging. On MRA, 7 of the 9 patients with follow-up had resolution of luminal narrowing (Fig $2 F$ and On-line Fig 3D), 1 

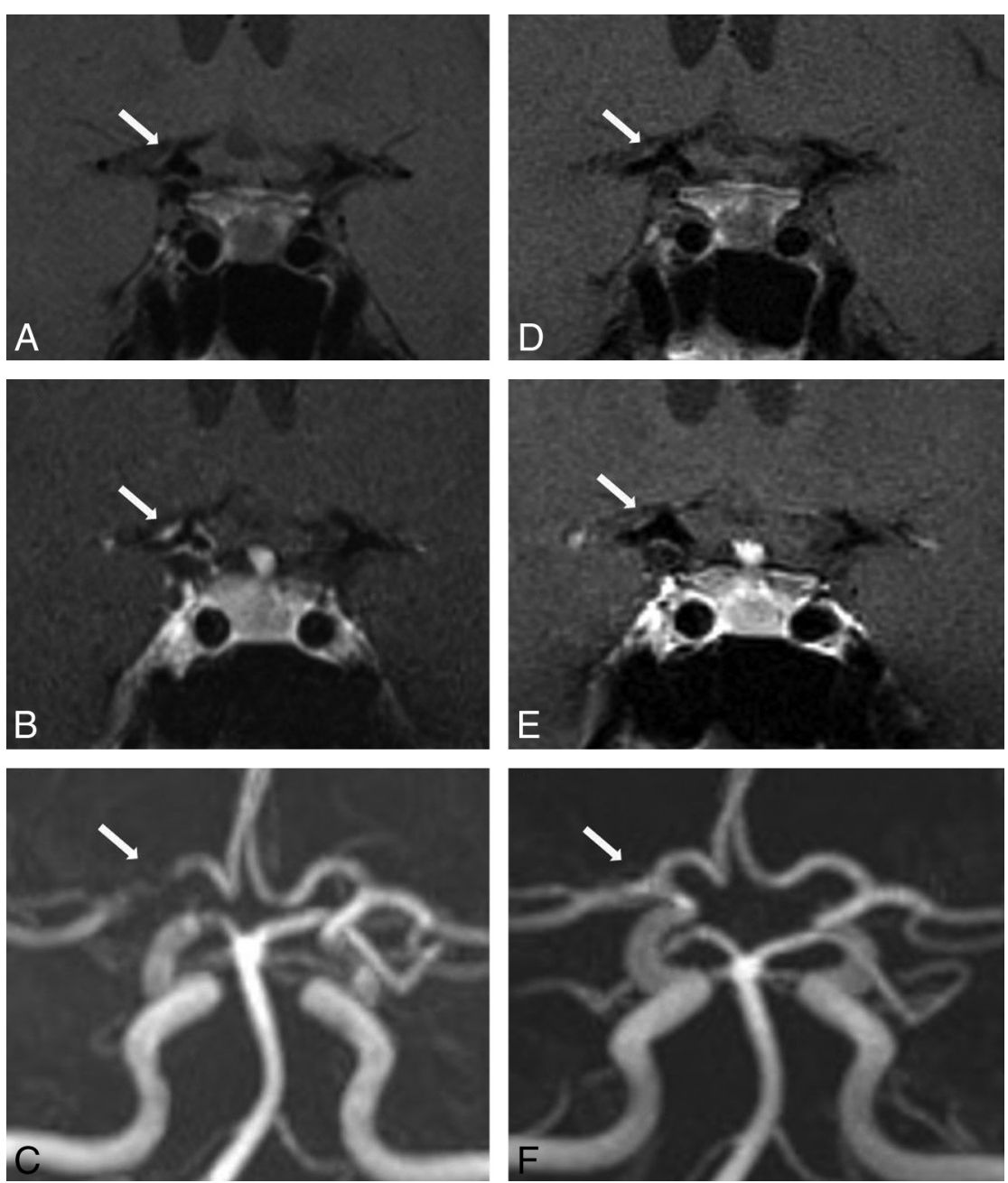

FIG 1. A 41-year-old woman with CNS vasculitis. 3T-HRMRI pre- and postgadolinium $(A$ and $B)$ T1-weighted arterial wall coronal images of terminal supraclinoid ICA and proximal M1 with strong smooth, concentric wall enhancement and thickening. C, MRA shows stenosis of the lumen and narrowing of M1 and the terminal supraclinoid ICA. Follow-up at 12 months. D and E, Pre- and post-gadolinium TIWI with near resolution of enhancement on coronal view. F, MRA with interval resolution of luminal narrowing and stenosis.

patient still had improved diffuse irregularity, and 1 patient had narrowing.

\section{DISCUSSION}

With the advent of higher field strength magnets and higher spatial resolution imaging, arterial wall characteristics of thickening and enhancement have been described for intracranial arterial diseases. ${ }^{6-9}$ In this study, we describe distinct arterial wall findings for RCVS and CNS vasculitis at initial diagnostic HRMRI. In addition, we describe a long period of stable persistent arterial wall findings for CNS vasculitis compared with a shorter period of resolution of vessel wall findings for RCVS at follow-up.

There has been notable interest in understanding the clinical manifestations and underlying pathophysiology of reversible cerebral vasoconstriction syndrome since it was coined by Calabrese et al. ${ }^{1}$ The syndrome describes a cluster of self-limiting disease entities that present with sudden recurrent thunderclap headaches, with or without additional neurologic symptoms and less commonly ischemic or hemorrhagic stroke resulting from persistent cerebral arterial vasoconstriction. ${ }^{1-4}$ The definitive patho- physiology of the cerebral artery vasoconstriction or the relation of symptoms to vasoconstriction is not yet clearly understood. ${ }^{3,4,13}$ Clinically, RCVS is known to closely resemble CNS vasculitis, which also presents with headache and neurologic symptoms from cortical and/or subcortical infarctions. ${ }^{14}$ Straightforward cases of RCVS may be distinguished from CNS vasculitis by using established RCVS criteria. ${ }^{2}$ On the other hand, with clinically ambiguous cases, diagnosis is often further confounded by similar angiographic findings. On DSA, the nonspecific characteristic "beading" pattern of segmental dilation and narrowing of multiple cerebral arteries seen in CNS vasculitis is identical to the multifocal alternating vasoconstriction and dilation/normal caliber vessels seen in RCVS. ${ }^{2,14-16}$ Likewise, both cerebrovascular entities share identical findings of segmental vessel stenosis on both MRA and CTA. ${ }^{1,3}$ Reversibility of angiographic findings for RCVS known to occur after 3 months may provide some radiographic differentiation for CNS vasculitis. ${ }^{2}$ However, delay in diagnosis may preclude immunosuppressive treatment critical for CNS vasculitis disease management to prevent the debilitating long-term sequelae of stroke; or immunosuppressive treatment, wrongly administered due to incorrect diagnosis, may worsen the prognosis for RCVS., ${ }^{2,17}$

On review of the literature, only 1 study to date has characterized the HRMRI vessel wall findings for RCVS and contrasted it with CNS vasculitis. Mandell et al, ${ }^{7}$ compared HRMRI artery wall characteristics for RCVS and CNS vasculitis. Their study, with findings similar to ours, clearly differentiated distinct wall characteristics for both diseases. However, like other HRMRI studies, ${ }^{6,7,18-20}$ it is limited in that it does not further characterize temporal wall patterns during the course of disease activity. Using HRMRI of 3 patients with RCVS and 4 patients with CNS vasculitis, the group described wall thickening and enhancement in CNS vasculitis, wall thickening with absent enhancement in RCVS, and similar findings of moderate-to-severe narrowing on TOF-MRA for both RCVS and CNS vasculitis. On follow-up TOF-MRA, their study showed persistence of the initial luminal findings for CNS vasculitis during a median of 15 months (range, 6-36 months) and resolution of RCVS after a median of 1.5 months (range, 1-3 months). This length of time for CNS vasculitis and RCVS on TOF-MRA was fairly consistent with the length of time for CNS vasculitis and RCVS on TOF-MRA in our study. However, their study did not describe follow-up HRMRI for CNS vasculitis and RCVS. 

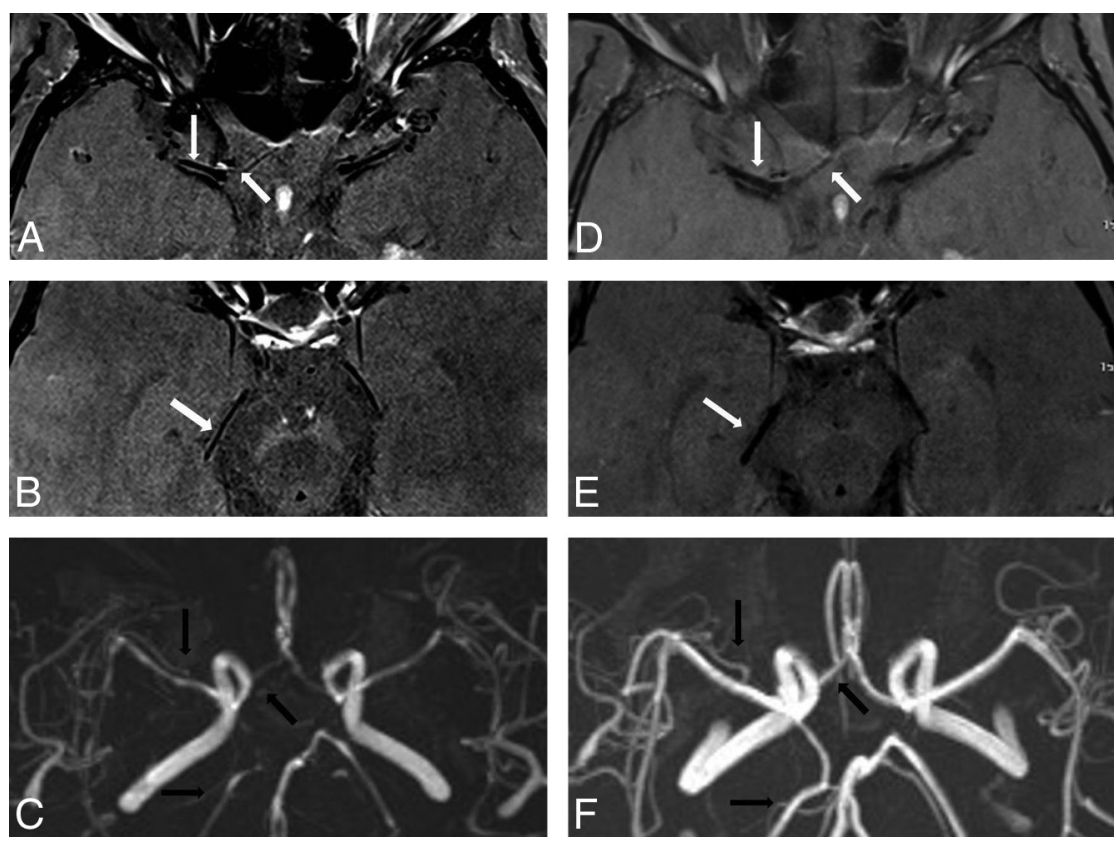

FIG 2. A 61-year-old woman with RCVS. 3T-HRMRI postgadolinium T1-weighted arterial wall with fat suppression and saturation band. $A$ and $B$, Axial images in the same planes show bilateral $A l, M l$, and P2 with diffuse uniform wall thickening and wall narrowing with mild enhancement. C, MRA shows corresponding bilateral $\mathrm{Al}, \mathrm{Ml}$, posterior communicating artery, and $\mathrm{P} 2$ narrowing. Follow-up vessel wall imaging at 3 months. $D$ and $E$, Postgadolinium axial imaging with interval near resolution of uniform wall thickening and wall narrowing in the $\mathrm{Al}$ and $\mathrm{Ml}$ segments and resolution of uniform wall thickening and wall narrowing in the P2 segment. F, Follow-up MRA with resolution of luminal narrowing with patent bilateral $\mathrm{A} 1, \mathrm{Ml}$, posterior communicating artery, and $\mathrm{P} 2$.

We observed, in our larger study population, that HRMRI findings were remarkably different for CNS vasculitis and RCVS at initial imaging and at follow-up. In the earlier disease course of RCVS, the pattern of diffuse uniformity with or without mild enhancement was continuous throughout the entire wall of the diseased vessel. This finding was clearly distinct in the short segments of wall thickening with concentric or eccentric enhancement in a unilateral distribution or bilateral artery distribution localized to 1 or multivessel segments seen in CNS vasculitis. The postgadolinium pattern of smooth, concentric wall enhancement and thickening was seen in $70 \%$ of patients, and eccentric wall enhancement and thickening was seen in $30 \%$ of patients.

In addition, CNS vasculitis had greater enhancement compared with mild enhancement if present in RCVS. On follow-up HRMRI for RCVS, there was early resolution of initial findings within a median of 3 months for all patients compared with CNS vasculitis, with a longer median of 7 months for resolution and 13.5 months for stable persistence of enhancement. These follow-up vessel wall and lumen findings on HRMRI appear compatible with the respective cerebrovascular disease course of RCVS and CNS vasculitis. The vessel wall findings of diffuse luminal narrowing and wall thickening with a short reversal time to normal vessel morphology appear consistent with the disease course of RCVS. ${ }^{1}$ The HRMRI findings of short, scattered segments of vessel wall enhancement and thickening in a multivessel distribution appear compatible with the pathologic course of CNS vasculitis. $^{2}$

To our knowledge, on review of the literature, there are no studies that describe follow-up vessel wall imaging characteristics for RCVS, while 2 studies attempt to describe follow-up for CNS vasculitis. ${ }^{21,22}$ Pfefferkorn et $\mathrm{al}^{22}$ in their HRMRI study, described moderate-to-strong enhancement in 4 patients with diagnoses of large-artery primary angiitis of the CNS. After immunosuppressive therapy, follow-up HRMRI showed that 2 patients had stable enhancement for 2 months and 2 patients had resolution of enhancement by 6 months. The second study, a case report by Saam et al, ${ }^{22}$ reported strong vessel wall enhancement in multiple arteries of a patient with intracranial arteritis. Follow-up imaging at 3 months showed a slight decrease in enhancement, and at 6 months, there was further substantial decrease with some persistent enhancement. In our study, the wall characteristics of smooth, concentric enhancement and thickening were stable for a follow-up median of 13.5 months in 4 patients. In contrast, in 2 patients, there was complete resolution of the smooth, concentric wall enhancement and thickening after a mean of 7 months. The findings in our study compared with the other authors ${ }^{21,22}$ demonstrate a greater period of wall thickening and enhancement for CNS vasculitis.

There were several limitations to our study. This retrospective study was conducted by using a small number of patients. However, it may represent the largest follow-up HRMRI comparison series of RCVS and CNS vasculitis to date. We are uncertain whether the mild wall enhancement seen in the 4 patients with RCVS is indeed inflammation because this has been refuted. ${ }^{7}$ However, inflammation has been reported in persistent vasoconstriction from vasospasm in subarachnoid hemorrhage. ${ }^{23}$ Larger prospective studies are need to further investigate and clarify these wall findings of enhancement in RCVS. We are also uncertain as to why there were variations in the length of time of enhancement, hence inflammation, in the CNS vasculitis group. These variations during follow-up may be the result of remission or flare-up of disease in response to therapy. We were unable to clearly determine the cause due to the retrospective nature of our study. However, in studies of extracranial vasculitis, wall enhancement has been shown to be strongest in the clinically acute symptomatic stage of the disease and with time with minimal or no enhancement with steroids. ${ }^{24,25}$ We postulated that similar to extracranial vasculitis, postgadolinium wall enhancement as a marker for inflammation in vasculitis would not be indefinite; rather, there would be a gradual loss of enhancement as the inflammation subsides. However, we found there was stable enhancement for many months in some patients in the CNS vasculitis patient group while on chronic immunosuppressive therapy. We are uncertain whether this stable enhancement with treatment is indicative of continuous active inflammatory activity, poor response to treatment, inadequate immunosuppressive therapy, or persistent con- 
trast leakage into the vessel wall after initial inflammatory damage of the endothelial lining. We also cannot ascertain whether response to treatment was a confounder that accounts for differences in enhancement in the CNS vasculitis group. This study also warrants a larger prospective study to answer questions as to the relationship of HRMRI findings and corresponding flare imaging or remission of disease activity with treatment.

\section{CONCLUSIONS}

Our study shows distinguishing HRMRI wall patterns for RCVS and CNS vasculitis at initial diagnostic imaging and at follow-up. It may also show that the ability of HRMRI to visualize the wall and lumen of large intracranial vessels can potentially increase the specificity of imaging to diagnose disparate vascular diseases with similar angiographic findings.

\section{ACKNOWLEDGMENTS}

We thank Craig Lisicki, MR Imaging Education Specialist at the Cleveland Clinic, for his technical assistance.

Disclosures: Ferdinand Hui-UNRELATED: Consultancy: MicroVention, Payment for Lectures (including service on Speakers Bureaus): MicroVention; Stock/Stock Options: Blockade Medical.

\section{REFERENCES}

1. Calabrese LH, Dodick DW, Schwedt TJ, et al. Narrative review: reversible cerebral vasoconstriction syndromes. Ann Intern Med 2007;146:34-44

2. Singhal AB, Hajj-Ali RA, Topcuoglu MA, et al. Reversible cerebral vasoconstriction syndromes: analysis of 139 cases. Arch Neurol 2011;68:1005-12

3. Ducros A, Boukobza M, Porcher $\mathrm{R}$, et al. The clinical and radiological spectrum of reversible cerebral vasoconstriction syndrome: a prospective series of 67 patients. Brain 2007;130:3091-101

4. Ducros A. Reversible cerebral vasoconstriction syndrome. Lancet Neurol 2012;11:906-17

5. Chen SP, Fuh JL, Wang SJ, et al. Magnetic resonance angiography in reversible cerebral vasoconstriction syndromes. Ann Neurol 2010;67:648-56

6. Swartz RH, Bhuta SS, Farb RI, et al. Intracranial arterial wall imaging using high-resolution 3-Tesla contrast-enhanced MRI. Neurology 2009;72:627-34

7. Mandell DM, Matouk CC, Farb RI, et al. Vessel wall MRI to differentiate between reversible cerebral vasoconstriction syndrome and central nervous system vasculitis: preliminary results. Stroke 2012;43:860-62

8. Kim YS, Lim SH, Oh KW, et al. The advantage of high-resolution MRI in evaluating basilar plaques: a comparison study with MRA. Atherosclerosis 2012;224:411-16
9. Vergouwen MD, Silver FL, Mandell DM, et al. Eccentric narrowing and enhancement of symptomatic middle cerebral artery stenoses in patients with recent ischemic stroke. Arch Neurol 2011;68:338-42

10. Headache Classification Subcommittee of the International Headache Society. The International Classification of Headache Disorders: 2nd ed. Cephalalgia 2004;24:9-160

11. Calabrese LH, Mallek JA. Primary angiitis of the central nervous system: report of 8 new cases, review of the literature, and proposal for diagnostic criteria. Medicine (Baltimore) 1988;67:20-39

12. Ferguson GG, Eliasziw M, Barr HW, et al. The North American Symptomatic Carotid Endarterectomy Trial: surgical results in 1415 patients. Stroke 1999;30:1751-58

13. Ducros A, Fiedler U, Porcher R, et al. Hemorrhagic manifestations of reversible cerebral vasoconstriction syndrome: frequency, features, and risk factors. Stroke 2010;41:2505-11

14. Koopman K, Uyttenboogaart M, Luijckx GJ, et al. Pitfalls in the diagnosis of reversible cerebral vasoconstriction syndrome and primary angiitis of the central nervous system. Eur J Neurol 2007;14: 1085-87

15. Gerretsen P, Kern RZ. Reversible cerebral vasoconstriction syndrome or primary angiitis of the central nervous system? Can J Neurol Sci 2007;34:467-77

16. Demaerel P, De Ruyter N, Maes F, et al. Magnetic resonance angiography in suspected cerebral vasculitis. Eur Radiol 2004;14:1005-12

17. French KF, Hoesch RE, Allred J, et al. Repetitive use of intra-arterial verapamil in the treatment of reversible cerebral vasoconstriction syndrome. J Clin Neurosci 2012;19:174-76

18. Küker W, Gaertner S, Nagele $T$, et al. Vessel wall contrast enhancement: a diagnostic sign of cerebral vasculitis. Cerebrovasc Dis 2008;26:23-29

19. Aoki S, Hayashi N, Abe O, et al. Radiation-induced arteritis: thickened wall with prominent enhancement on cranial MR images report of five cases and comparison with 18 cases of Moyamoya disease. Radiology 2002;223:683-88

20. Pfefferkorn T, Schuller U, Cyran C, et al. Giant cell arteritis of the basal cerebral arteries: correlation of MRI, DSA, and histopathology. Neurology 2010;74:1651-53

21. Pfefferkorn T, Linn J, Habs M, et al. Black blood MRI in suspected large artery primary angiitis of the central nervous system. J Neuroimaging 2013;23:379-83

22. Saam T, Habs M, Pollatos O, et al. High-resolution black-blood contrast-enhanced $\mathrm{T} 1$ weighted images for the diagnosis and follow-up of intracranial arteritis. Br J Radiol 2010;83:e182-84

23. Carr KR, Zuckerman SL, Mocco J. Inflammation, cerebral vasospasm, and evolving theories of delayed cerebral ischemia. Neurol Res Int 2013;2013:506584

24. Bley TA, Wieben O, Uhl M, et al. High-resolution MRI in giant cell arteritis: imaging of the wall of the superficial temporal artery. AJR Am J Roentgenol 2005;184:283-87

25. Bley TA, Wieben $\mathrm{O}$, Leupold $\mathrm{J}$, et al. Images in cardiovascular medicine: magnetic resonance imaging findings in temporal arteritis. Circulation 2005;111:e260 